\title{
DRUG RESISTANCE AND BIOFILM PRODUCTION AMONG PSEUDOMONAS AERUGINOSA CLINICAL ISOLATES IN A TERTIARY GARE HOSPITAL OF NEPAL
}

\author{
Shrestha R, Nayak N, Bhatta DR, Hamal D, Subramanya SH, Gokhale S
}

Department of Microbiology, Manipal College of Medical Sciences, Pokhara, Nepal

\begin{abstract}
Clinical isolates of Pseudomonas aeruginosa often exhibit multidrug resistance due to their inherent ability to form biofilms. Drug resistance in Ps. aeruginosa is a major clinical problem, especially in the management of patients with nosocomial infections and those admitted to ICUs with indwelling medical devices. To evaluate the biofilm forming abilities of the clinical isolates of Ps. aeruginosa and to correlate biofilm formation with antibiotic resistance. A total of 90 consecutive isolates of Ps. aeruginosa obtained from various specimens collected from patients visiting the Manipal Teaching Hospital, Pokhara, Nepal between January 2018 - October 2018 were studied. Isolates were identified by standard microbiological methods. Antibiotic susceptibility testing was performed by Kirby-Bauer disc diffusion method. All the isolates were tested for their biofilm forming abilities by employing the tissue culture plate assay. Of the 90 Ps. aeruginosa isolates, maximum i.e $42(46.6 \%)$ were from patients in the age group of > 50 years. Majority (30; 33.3\%) of the isolates were obtained from sputum samples. However, percentage isolation from other specimens like urine, endotracheal tube (ETT), pus, eye specimens and blood were $18.9 \%, 16.7 \%, 16.7 \%, 7.8 \%$ and $6.7 \%$ respectively. All the isolates were sensitive to polymixin B and colistin, $91.1 \%$ of the organisms were sensitive to imipenem, and more than $80 \%$ to aminoglycosides ( $80 \%$ to gentamicin, $83.3 \%$ to amikacin). A total of 29 (32.2\%) organisms were biofilm producers. Maximum numbers of biofilm producing strains were obtained from ETT (8 of 15; 53.3\%), pus (8 of 15; 53.3\%) and blood ( 2 of 6; 33.3\%) i.e from all invasive sites. None of the isolates from noninvasive specimens such as conjunctival swabs were biofilm positive. Significantly higher numbers of biofilm producers (23 of 29; 79.3\%) were found to be multidrug resistant as compared to non-biofilm (6 of $61 ; 9.8 \%$ ) producers ( $\mathrm{p}=0.000)$. Ps. aeruginosa colonization leading to biofilm formation in deep seated tissues and on indwelling devices is a therapeutic challenge as majority of the isolates would be recalcitrant to commonly used antipseudomonal drugs. Effective monitoring of drug resistance patterns in all Pseudomonas clinical isolates should be a prerequisite for successful patient management.
\end{abstract}

\section{KEYWORDS}

Pseudomonas aeruginosa, antibiogram, biofilm, multi-drug-resistance

\section{CORRESPONDING AUTHOR}

Dr. Rajani Shrestha

Lecturer,

Department of Microbiology

Manipal College of Medical Sciences

Pokhara, Nepal

Email: rajani_sth7@hotmail.com 


\section{INTRODUCTION}

The gram-negative bacterium, Pseudomonas aeruginosa belongs to a vast genus of obligate aerobic, non-fermenting, saprophytes, which are present in water, soil and on plants. ${ }^{1}$ Moreover, Ps. aeruginosa can be frequently isolated from tap water. In its natural habitat, this organism is endowed with weak pathogenic potential. However, its profound ability to survive on inert materials, its minimal nutritional requirement, tolerance to a wide variety of physical conditions and relative resistance to several antimicrobial agents and antiseptics, contribute enormously to its ecological success and its role as an effective opportunistic pathogen. ${ }^{2}$ Ps. aeruginosa is a notoriously difficult organism to control with antibiotics or disinfectants and has become increasingly recognized as an emerging opportunistic pathogen of clinical relevance.

Several different epidemiological studies tracked its emergence as multi-drug-resistant Ps. aeruginosa (MDRPA) strains among the clinical isolates, ${ }^{3}$ and this organism was accounted for a significant proportion of nosocomial infections. ${ }^{4}$ MDRPA are often isolated from patients suffering from nosocomial infections, particularly those in the intensive care unit (ICU). ${ }^{5}$ Thus, infections caused by Ps. aeruginosa especially in ICU patients are problematic because the organism apart from being inherently resistant to many drug classes, is able to acquire resistance to many effective antimicrobial drugs ${ }^{2}$ and therefore infections caused by Ps. aeruginosa are frequently life threatening and difficult to treat. ${ }^{6,7}$ Such multidrug resistance could be due to the slowly growing state of $P$. aeruginosa in the deeper layers of thick biofilms, which the organism has a tendency to form in many in vivo situations. ${ }^{8}$ Biofilm cells have less access to antibiotics due to the impaired diffusion of antibiotic molecules through the biofilm matrix. Moreover, there is increased horizontal gene transfer among biofilm organisms, with high plasmid transfer rates, which aggravates the problem of drug resistance further. ${ }^{8}$ As organisms growing in a biofilm are more resistant to antimicrobial agents than free floating planktonic cells, high antimicrobial concentrations (1000 fold higher) are often required to inactivate cells growing in the interior of a biofilm..$^{9}$ Thus, the aim of this study was to evaluate the biofilm forming abilities of the clinical isolates of Ps. aeruginosa and to determine the correlation of biofilm formation with multidrug resistance.

\section{MATERIALS AND METHODS}

A total of 90 consecutive isolates of Ps. aeruginosa obtained from various clinical specimens such as pus/wound swab, blood, sputum, urine, endotracheal tube (ETT) and conjunctival swabs collected from patients visiting the Manipal Teaching Hospital, Pokhara, Nepal between January 2018 and October 2018 were studied. Organisms were identified by standard microbiological techniques. ${ }^{10}$ Antimicrobial sensitivity testing was performed on Mueller-Hinton agar plates with commercially available antibiotic discs (Hi-media, Mumbai, India) using Kirby Bauer disc diffusion technique ${ }^{11}$ and interpreted as per the guidelines of CLSI. The antibiotic discs (conc.) used were piperacillin/tazobactum (100/10mcg), ceftazidime (30 mcg), ciprofloxacin ( $5 \mathrm{mcg})$, amikacin (30 mcg), imipenem (10mcg), cefepime (30 mcg), polymyxin B (300units), gentamicin (10 mcg), and colistin (10mcg). Ps. aeruginosa strain ATCC 15442 was used as the control. Isolates showing resistance to at least one agent in three or more antimicrobial categories based on the guidelines recommended by joint initiative of the European Centre for Disease Prevention and Control (ECDC) and the Centers for Disease Control and Prevention (CDC), ${ }^{12}$ were leveled as MDRPA.

All these isolates were tested for their biofilm forming abilities by employing the tissue culture plate assay, earlier described by Christensen et al. ${ }^{13}$ Briefly, a single colony of $P$ s. aeruginosa was grown overnight at $37^{\circ} \mathrm{C}$ in $2 \mathrm{~mL}$ of trypticase soy broth (TSB). The bacterial culture was then diluted (1:100) with sterile fresh TSB. Each well of a 96-well flatbottomed polystyrene tissue culture plate was filled with $200 \mu \mathrm{L}$ of the diluted culture. The plate was incubated for $24 \mathrm{~h}$ at $37^{\circ} \mathrm{C}$. The content of each well was aspirated carefully and then plate was gently washed with phosphate buffer saline (PBS), pH 7.2 in order to remove free-floating bacteria. Adherent bacteria were fixed with 99\% methanol for 10-15 min. The content of the plate was decanted, allowed to dry and stained for 10 min with $1 \%$ crystal violet (CV). Excess stain was removed by rinsing the plate with tap water. Optical density (OD) of stained adherent biofilm was measured by using a microELISA reader (Human) at a wavelength of $570 \mathrm{~nm}$. Ps. aeruginosa ATCC strain 15442 was used as the positive control. Ten wells stained exactly in the same manner, but without any organism (sterile broth only) served as the negative control (blanks). The interpretation of biofilm production was made as per the criteria of Stepanovic et al, ${ }^{14}$ and the cut off was calculated as 3XSD above the mean of the ten blanks.

\section{RESULTS}

A total of 90 Ps. aeruginosa isolates were identified during the study period. Distribution of Ps. aeruginosa isolates with respect to age and gender showed that 54 isolates were from male patients and 36 were from females. Irrespective of differences in gender, maximum strains were isolated in the age group of $>50$ years.

Rates of isolation of Ps. aeruginosa from various clinical specimens have been shown in table 1 . Maximum numbers of isolates $(30 ; 33.3 \%)$ were obtained from sputum. However, percentage isolation from other specimens like urine, endotracheal tube (ETT), pus, eye specimens and blood were $18.9 \%, 16.7 \%, 16.7 \%, 7.8 \%$ and $6.7 \%$ respectively. 


\begin{tabular}{|lcc|}
\hline \multicolumn{2}{|c|}{$\begin{array}{c}\text { Table 1: Pseudomonas aeruginosa isolates } \\
\text { from various clinical specimens. }\end{array}$} \\
\hline Sources of specimen & $\mathbf{n}$ & $\%$ \\
Eye specimens & & 7.8 \\
(conjunctival swab) & 7 & 6.7 \\
Blood & 6 & 18.9 \\
Urine & 17 & 33.3 \\
Sputum & 30 & 16.7 \\
Pus/wound & 15 & 16.7 \\
ETT & 15 & $\mathbf{1 0 0 . 0}$ \\
Total & $\mathbf{9 0}$ & \\
\hline
\end{tabular}

Overall antibiotic sensitivity pattern of the isolates are shown in Fig. 1. Whereas 100\% of the organisms were sensitive to both polymixin B and colistin. Ninety one percent were sensitive to imipenem, and more than $80 \%$ to aminoglycosides $(80 \%$ to gentamicin, $83.3 \%$ to amikacin). Sensitivity
(2 of 6; 33.3\%) i.e all from invasive sites. Contrary to this none of the isolates from noninvasive specimens such as conjunctival swabs were shown to be biofilm positive.

\begin{tabular}{|lccc|}
\hline \multicolumn{3}{|c|}{ Table 2: Biofilm producer and non-biofilm } \\
producer organisms obtained from various \\
clinical specimens. \\
Specimens & $\begin{array}{c}\text { Non } \\
\text { Biofilm } \\
\text { producer (\%) }\end{array}$ & $\begin{array}{c}\text { Producer } \\
\text { (\%) }\end{array}$ & Total \\
Eye samples & $7(100 \%)$ & $0(0 \%)$ & 7 \\
Blood & $4(66.7 \%)$ & $2(33.3 \%)$ & 6 \\
Urine & $11(74.85 \%)$ & $6(25.2 \%)$ & 17 \\
Sputum & $25(83.4 \%)$ & $5(16.6 \%)$ & 30 \\
Pus & $7(46.7 \%)$ & $8(53.3 \%)$ & 15 \\
ETT & $7(46.7 \%)$ & $8(53.3 \%)$ & 15 \\
Total & $61(67.7 \%)$ & $(32.3 \%)$ & 90 \\
\hline
\end{tabular}

$P$ value $=0.025$

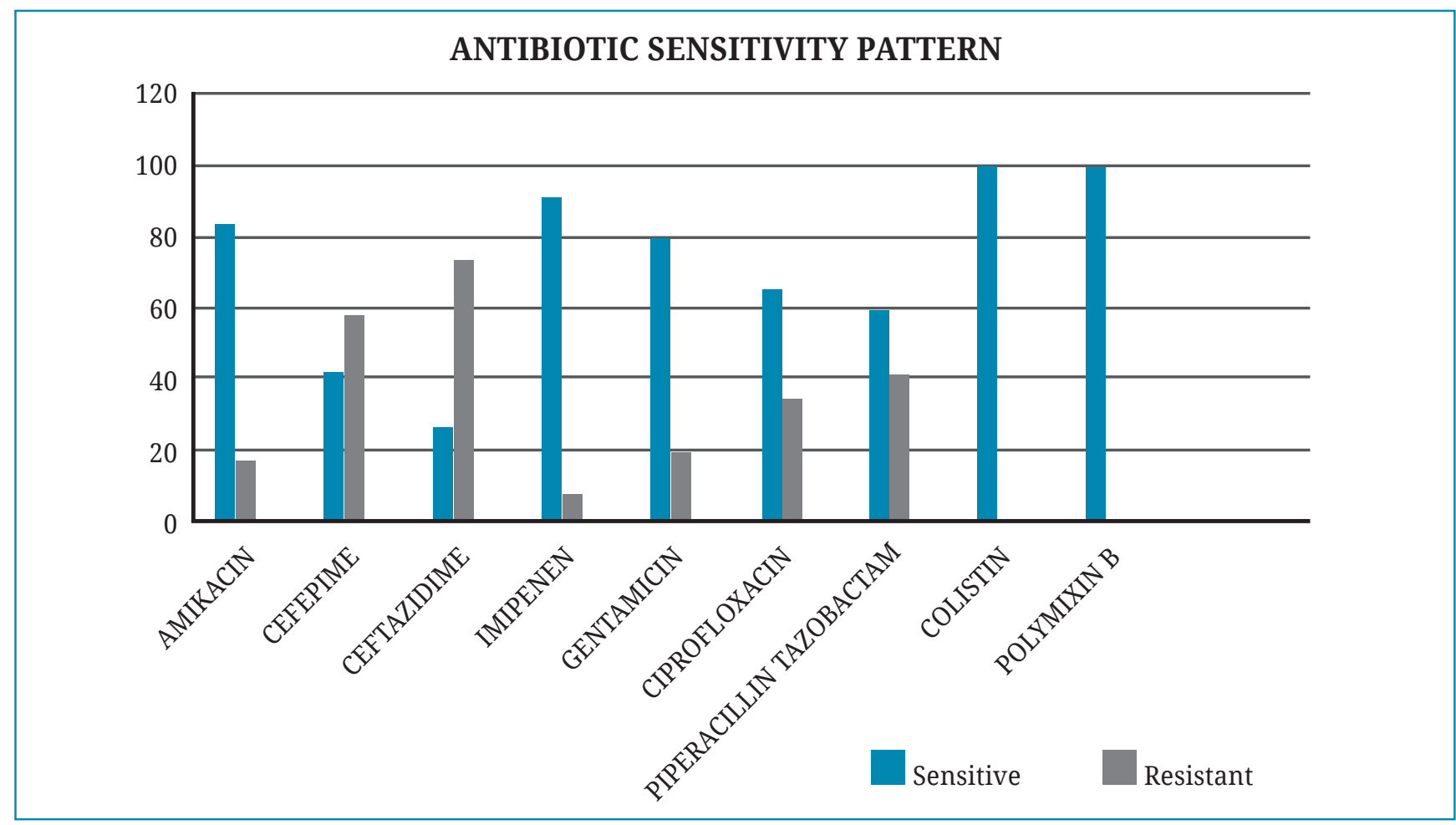

Fig. 1: Antibiotic sensitivity pattern of the isolates.

patterns were less promising against ciprofloxacin, piperacillin tazobactam, cefepime and ceftazidime; the rates of sensitivities being $65.5 \%, 58.9 \%, 42.2 \%$ and $26.7 \%$ respectively.

Biofilm forming ability determined by tissue culture plate method revealed that $29(32.2 \%)$ of the 90 isolates were biofilm producers. Distribution of biofilm producing organisms according to their sources of isolation has been depicted vide table 2 . It was interesting to note that maximum numbers of biofilm producing organisms were obtained from ETT (8 of 15; 53.3\%), pus (8 of $15 ; 53.3 \%$ ) and blood
Table 3: Correlation between biofilm formation and multidrug resistance (MDR)

\begin{tabular}{|lccc|}
$\begin{array}{l}\text { Biofilm } \\
\text { production }\end{array}$ & $\begin{array}{c}\text { MDR } \\
\text { n (\%) }\end{array}$ & $\begin{array}{c}\text { No MDR } \\
\text { n (\%) }\end{array}$ & Total \\
Producer & $23(79.3)$ & $6(20.7)$ & 29 \\
Non-producers & $6(9.8)$ & $55(90.2)$ & 61 \\
Total & $\mathbf{2 9 ( 3 2 . 2 )}$ & $\mathbf{6 1 ( 6 7 . 8 )}$ & $\mathbf{9 0}$ \\
\hline
\end{tabular}

$\mathrm{P}=0.000$ 
Table 3 shows the correlation between biofilm formation and multi-drug resistance among the isolates. Higher number of biofilm producers (23 of 29; 79.3\%) were found to be multidrug resistant as compared to only 6 of the 61 ; $(9.8 \%)$ nonbioflim producers. This difference was found to be statistically significant $(\mathrm{p}<0.001)$ commonly isolated nosocomial pathogen accounting for $10.1 \%$ of all hospital acquired infections. ${ }^{3}$

There are limited number of antimicrobial agents with reliable activity against $P$ s. aeruginosa including anti-pseudomonal penicillins and cephalosporins, carbepenems, aminoglycosides

Table 4: Resistance patterns in relation to biofilm production

\begin{tabular}{|lccc|}
$\begin{array}{l}\text { Resistance to } \\
\text { antibiotics }\end{array}$ & \multicolumn{1}{c}{ Biofilm } & p value \\
Amikacin & Non producer (\%) (n-61) & Producer (\%) (n-29) & \\
Cefepime & $5(8.2)$ & $10(34.5)$ & $\mathbf{0 . 0 0 2}$ \\
Ceftazidime & $27(44.3)$ & $25(86.2)$ & $\mathbf{0 . 0 0 0}$ \\
Imipenem & $41(67.2)$ & $25(86.2)$ & 0.057 \\
Gentamicin & $2(3.3)$ & $6(20.7)$ & 0.007 \\
Ciprofloxacin & $6(9.8)$ & $13(44.9)$ & $\mathbf{0 . 0 0 0}$ \\
Piperacillintazobactam & $10(16.4)$ & $21(72.4)$ & $\mathbf{0 . 0 0 0}$ \\
Polymixin B & $15(24.6)$ & $22(75.4)$ & $\mathbf{0 . 0 0 0}$ \\
Colistin & $0(0)$ & $0(0)$ & - \\
\hline
\end{tabular}

Table 4 depicts the resistance patterns of biofilm producers vs. biofilm non-producers against individual antibiotics tested. Significantly higher numbers of biofilm producers were resistant to all the antibiotics except ceftazidime for which no significant difference in the resistance rates were detected among both the groups. However, all the organisms, irrespective of biofilm production, were found to be sensitive to both polymixin B and colistin.

\section{DISCUSSION}

Ps. aeruginosa is a leading cause of nosocomial infections, ranking only second among the gramnegative pathogens. ${ }^{2}$ Resistance to antimicrobials among Ps. aeruginosa is an increasing clinical problem and is a recognized public health threat.15

In cases of polymicrobial bacteremia, Ps. aeruginosa is the main cause of mortality and second most common bacterium causing sepsis in the intensive care unit. ${ }^{16,17}$ In addition, Ps. aeruginosa is one of the infuriating pathogens implicated in urinary tract infections (UTIs), burn and post-operative wound infections, lower respiratory tract infections (LRTIs), cystic fibrosis (CF), and ventilator associated pneumonia (VAP). ${ }^{16}$ According to CDC, the overall incidence of Ps. aeruginosa infection in the US hospitals averages about $0.4 \%$ (4 per 1000 discharges), and the bacterium is the fourth most and fluoroquinolones. However, Ps. aeruginosa clinical isolates show remarkable properties for the development of resistance, which is the reason for increased rate of mortality and morbidity due to infection by this organism.

We found multidrug resistance among 32.2\% (29/90) of our isolates. Other workers from Nepal reported $18.6 \%$ and $20.5 \%$ of the Pseudomonas isolates to be multidrug resistant (MDR). ${ }^{18,19}$ Yet lower rates of MDR were noted by studies outside Nepal..$^{20}$ Contrarily, higher resistance rates noted in our study as compared to others may be because the organisms in our investigation were isolated not only from blood but from other specimens like urine, sputum, pus as well. Obritsh et $a l,{ }^{3}$ however, were of the view that rates of isolation of MDRPA varied between $0.6-32 \%$, and this variation was dependent upon the geographical location and the type of study. ${ }^{3}$

Nevertheless, multidrug resistance among Ps. aeruginosa is a major clinical problem especially among ICU patients, and in those with CF and on various indwelling devices, who provide enough opportunity for biofilm production by this organism in the above clinical situations. ${ }^{21}$ Over and above, the pathogenesis of the majority of chronic Ps. aeruginosa infections is mainly due to the capacity of this organism to form biofilm, that imparts resistance to many antibiotics. Such biofilms embedded bacteria are difficult to eradicate by 
conventional antibiotic therapy because bacterial cells living inside biofilms are more tolerant towards antibiotics than their planktonic counterparts. ${ }^{22}$

Biofilms have an enormous impact on health care. According to a recent study, biofilm related infective conditions are estimated to be responsible for almost $65 \%$ of all nosocomial infections. ${ }^{23}$ Till date, there are scanty reports in the literature on the discovery and evaluation of any antibiofilm agent which could attenuate the pathogenicity of biofilm forming Ps. aeruginosa. ${ }^{22}$

In the above context, we extended our study further in order to find if there were any biofilm producers among the isolates in our series. Our results showed that $32.2 \%$ of the isolates were biofilm producers. Heydari et al $^{24}$ reported $43.5 \%$ of the Ps. aeruginosa isolates from patients in the burn care units to be biofilm producers. Worlitzh and colleagues ${ }^{25}$ demonstrated that Ps. aeruginosa formed robust biofilms in CF patients, which reflected persistence of the organism in the $\mathrm{CF}$ lesions. In yet another recent study, Gilperotin et $a l^{21}$ noted that biofilm formation on the surfaces of ETT was a frequent occurrence and was related to the pathogenesis of VAP allowing persistence of the organism in ETT biofilms, affecting the prognosis adversely.

We detected biofilm-producing Ps. aeruginosa isolates from $53.3 \%$ of the ETT, $53.3 \%$ of pus specimen, $33.3 \%$ of blood, $25.3 \%$ of urine and $16.6 \%$ of sputum samples. Interestingly, we observed that a significantly higher number of biofilm forming Ps. aeruginosa showed MDR (79.3\%), as compared to non-biofilm producers. Abidi et al $^{26}$ studied 22 $P s$. aeruginosa isolates and concluded that biofilm production was significantly higher among MDRPA isolates. Further, Nithyalakshmi et $a l^{27}$ showed a statistically significant association of MDR in bacteria with their biofilm activity and thus showed that $12(25 \%)$ of their 48 biofilm producing Ps. aeruginosa were multidrug resistant as compared to only $5(7.8 \%)$ of the 64 non-biofilm producers. Gurung et $a l^{28}$ also observed that $57 \%$ of biofilm producing Ps. aeruginosa were of the multidrug resistant phenotype.

Overall antibiotic sensitivity pattern of the isolates revealed that $91.1 \%$ of the isolates were sensitive to imipenem, $>80 \%$ to aminoglycosides, $65.6 \%$ to ciprofloxacin, $58.9 \%$ to pipearacillin tazobactam. Alarmingly, $73.3 \%$ of the organism exhibited resistance against ceftazidime, which is supposed to be the drug of choice against Ps. aeruginosa, often preferred by many clinicians because of its optimum antipseudomonal activity. Various other studies in the past documented that 69 to $89 \%$ of pseudomonas nosocomial isolates were resistant to ceftazidime, according to the views of the researchers, could be the outcome of frequent use of this drug in clinical practice. ${ }^{2,29,30}$
Much in agreement with the present study, several reports in the past evidenced that multidrug resistant strains of Ps. aeruginosa were uniformly susceptible to polymixin B. ${ }^{31,32}$ All the isolates in the present study were sensitive to polymixin B and colistin. Similar observations were noted by Kalaivoni et al $^{33}$ from India. However, high incidence of nephrotoxicity and neurotoxicity associated with the administration of these drugs could be a limitation for their use in clinical practice. ${ }^{34}$ In recent years there had been dramatic increase in the resistance rates shown by Ps. aeruginosa towards imipenem. Fortunately, our study documented only $8.9 \%$ of the isolates to be resistant to this drug, which was much lower compared to the resistance rates presented by a study conducted in Nepal. ${ }^{35,18}$ Kateete et al from Uganda reported $40 \%$ carbapenemase and 66\% metallo-betalactamase (MBL) producing carbapenem resistant Ps. aeruginosa. ${ }^{36}$

In our study, irrespective of differences in gender, maximum number of Pseudomonas isolates (46.6\%) were obtained from the patient in the age group of $>50$ years. Similar observations were made by Chandel et $a l^{37}$ who reported that $31 \%$ of all Pseudomonas isolates from different samples (pus, sputum, urine tracheal aspirate, BAL fluid, bile, catheter, high vaginal swab) were from patients who were more than 60 years of age. These findings are of clinical relevance, as patients in these age groups may have decreased immunity, invariably have other co morbidity conditions, and are more likely to undergo prolonged hospitalization with indwelling medical devices, making them vulnerable to develop biofilm related infections.

As is evident from the data provided vide table 4, higher number of biofilm producing organisms exhibited resistance to all antibiotics tested except ceftazidime. This signifies that patients, especially those belonging to the older age groups, who are more likely to have indwelling medical devices, and are prone to develop biofilm on these devices, would be recalcitrant to majority of the routinely used antimicrobial agents. ${ }^{21}$

In conclusion, our study emphasizes on the dynamic relationship between Ps. aeruginosa colonization, biofilm formation and development of serious life threatening deep-seated infections. Biofilm once formed are responsible for bacterial persistence and their impaired response to antimicrobial therapy. 


\section{REFERENCES}

1. Valles J, Mariscal D, Cortes $\mathrm{P}$ et al. Patterns of colonization by Pseudomonas aeruginosa in intubated patients: a 3-year prospective study of 1,607 isolates using pulsed-field gel electrophoresis with implications for prevention of ventilator-associated pneumonia. Intensive Care Med 2004; 30: 1768-75.

2. Gales AC, Jones RN, Turnidge J, Rennie R, Ramphal R. Characterisation of Pseudomonas aeruginosa isolates: occurrence rate, antimicrobial susceptibility pattern and molecular typing in the Global SENTRY antimicrobial surveillance pro- gram 1997-1999. Clin Infect Dis 2001; 32: S146-155.

3. Obritsch MD, Fish DN, MacLaren R, Jung R. Nosocomial infections due to multidrug-resistant Pseudomonas aeruginosa: epidemiology and treatment options. Pharmacotherapy 2005; 25: 1353-64.

4. Blanc DS, Petignat C, Janin B et al. Frequency and molecular diversity of Pseudomonas aeruginosa upon admission and during hospitalization: a prospective epidemiologic study. Clin Microbiol Infect 1998; 4: 242-7.

5. Tassios PT, Gennimata V, Spaliara-Kalogeropoulou $\mathrm{L}$ et al. Multiresistant Pseudomonas aeruginosa serogroup 0:11 outbreak in an intensive care unit. Clin Microbiol Infect 1997; 3: 621-8.

6. Poole K. Pseudomonas aeruginosa: Resistance to the max. Front Microbiol 2011; 2: 1-13.

7. Kerr KG, Snelling AM. Pseudomonas aeruginosa: a formidable and ever-present adversary. J Hosp Infect 2009; 73: 338-44.

8. Diaz MA, Hernandez JR, Martinez-Martinez L, Rodrifuez-Bano J, Pascual A. Extended-spectrumbetalactamase producing Escherichia coli and Klebsiella pneumoniae in Spanish hospitals: 2nd multicenter study (GEIHBLEE project, 2006). Enferm Infect Microbiol Clin 2009; 27: 503-10.

9. Stewart PS, Costerton J. Antibiotic resistance of bacteria in biofilms. Lancet 2001; 358: 135.

10. Forbes BA, Sham DF, Weissfeld AS. Bailey and Scott's diagnostic Microbiology, $10^{\text {th }}$ ed. New York: Mosby; 1998. p. $167-87$

11. Bauer KBR, Kerby WMM, Sherris JC, Turck. M. Antibiotic Susceptility testing by a standardized single disc method. Am J Clin Pathol 1966; 45: 493-6.

12. Magiorakos AP, Srinivasan A, Carey RB et al. Multidrug-resistant, extensively drug-resistant and pandrug- resistant bacteria: an international expert proposal for interim standard definitions for acquired resistance. Clin Microbiol Infect 2012; 18: 268-81.

13. Christensen GD, Simpson WA, Younger JJ et al. Adherence of coagulase-negative staphylococci to plastic tissue culture plates: a quantitative model for the adherence of staphylococci to medical devices. $J$ Clin Microbiol 1985; 22: 996- 1006.

14. Stepanovic S, Vukovic D, Hola V et al. Quantification of biofilm in microtiter plates: overview of testing conditions and practical recommendations for assessment of biofilm production by staphylococci. Acta Pathol Microbiol Immunol Scandinavia 2007; 115: 891-9.
15. Arruda EA, Marinho IS, Boulos M et al. Nosocomial infections caused by multiresistant Pseudomonas aeruginosa. Infect Control Hosp Epidemiol 1999; 20: 620-3.

16. Marra AR, Bar K, Bearman GM et al. Systemic inflammatory response syndrome in nosocomial bloodstream infections with Pseudomonas aeruginosa and Enterococcus species: comparison of elderly and nonelderly patients. J Am Geriatr Soc 2006; 54: 804-8.

17. Vincent JL, Sakr Y, Sprung CL et al. Sepsis in European inten- sive care units: results of the SOAP study. Crit Care Med 2006; 34: 344 - 53.

18. Mishra SK, Acharya J, Kattel HP, Koirala J, Rijal BP, Pokhrel BM. Metallo-beta-lactamase producing gram negative bacterial isolates. J Nepal Health Res Counc 2012; 10: 208-13.

19. Shrestha S, Amatya R, Adhikari RP. Prevalence and antibiogram of Pseudomonas aeruginosa isolated from clinical specimens in a Teaching Hospital, Kathmandu: Nepal Med Coll J 2015; 17: 132-5.

20. Traub WH, Scheidhauer R, Leonhard B, Bauer D. Surveillance of Pseudomonas aeruginosa in intensive care units: clusters of nosocomial cross-infection and encounter of a multiple-antibiotic resistant strain. Chemotherapy 1998; 44: 243-59.

21. Gil-Perotin S, Ramirez P, Marti V et al. Implications of endotracheal tube biofilm in ventilator-associated pneumonia response: a state of concept. Crit Care 2012, 16: R93

22. Furiga A, Lajoie B, El Hage S, Baziard G, Roques C. Impairment of Pseudomonas aeruginosa Biofilm Resistance to Antibiotics by Combining the Drugs with a New Quorum-Sensing Inhibitor. Antimicrobial Agents Chemother 2016; 60: 1677-86.

23. Potera C. Forging a link between biofilms and disease. Science 1999; 283: 1837-9.

24. Heydari S, Eftekhar F. Biofilm formation and $\beta$ lactamase production in burn isolates of Pseudomonas aeruginosa. Jundishapur J Microbiol. 2015 Mar 21; 8: e15514.

25. Worlitzsch D, Tarran R, Ulrich M, Schwab U, Cekici A, Meyer KC et al. Effects of reduced mucus oxygen concentration in airway Pseudomonas infections of cystic fibrosis patients. J Clin Invest 2002; 109: 317-25.

26. Abidi SH, Sherwani SK, Siddiqui TR, Bashir A, Kazmi SU. Drug resistance profile and biofilm forming potential of Pseudomonas aeruginosa isolated from contact lenses in karachi Pakistan. BMC Opthalmol 2013; 13: 57.doi:10.1186/1471-2415-13-57.

27. Nithyalakshmi. J, Akila. K, Mohanakrishnan. K and Smanthi G. Evaluation of resistance profile of Pseudomonas aeruginosa with reference to biofilm production-An emerging challenge. IOSR-J Dental Med Sci 2015; 14: 13-8.

28. Gurung J, Khyriem AB, Banik A, Lyngdoh WV, Choudhury B and Bhattacharya P. Association of biofilm production with multidrug resistance among clinical isolates of Acinetobacter baumannii and Pseudomonas aeruginosa from intensive care unit. Indian J Crit Care Med 2013; 17: 214-8. 
29. Srinivas B, Lalitha Devi D, Narasinga Rao B. A prospective study of Pseudomonas aeruginosa and its antibiogram in a Teaching Hospital of Rural setup. $J$ Pharm Biomed Sci 2012; 22: 1-5.

30. Tahira M, Mohammed AM, Galnaz K, Mustafa K. Pseudomonas aeruginosa in chronic suppurative otitis media: sensitivity spectrum against various antibiotics in Karachi. J Ayub Med Coll Abbottabad 2009; 21: 120-3.

31. Koomanachai P, Tiengrim S, Kiratisin P, Thamliktikil V. Efficacy and safety of colistin (colistimethate sodium) for therapy of infections caused by multidrug resistant Pseudomonas aeruginosa and Acinetobacter baumannii in Siriraj Hospital, Bangkok, Thailand. Int'l J Infect Dis 2007; 5: 402-26.

32. Walsh TR, Toleman MA, Poirel L, Nordmann P. Metallo-beta-lactamases:the quiet before the storm? Clin Microbiol Rev 2005: 18; 306-25.

33. Kalaivani R, Shashikala P, Sheela Devi C, Prashanth K, Saranathan R. Phenotypic assays for detection of ESBL and MBL producers among the clinical isolates of multidrug resistant Pseudomonas aeruginosa from a tertiary care hospital. Int'l J Cur Res Rev 2013; Sep; 05: 28-35.

34. Falagas ME, Kaisiakov SK. Toxicity of polymixins: a systematic review of the evidence from old and recent studies. Crit Care 2006; 10: R27. doi: 10.1186/ cc3995.

35. Shrestha RK, Dahal RK, Mishra SK et al. Ventilator Associated Pneumonia in Tertiary Care Hospital, Maharajgunj, Kathmandu, Nepal. J Inst Med 2013; 35: 21-8.

36. Kateete DP, Nakanjako R, Namugenyi J, Erume J, Joloba ML, Najjuka CF. Carbapenem resistant Pseudomonas aeruginosa and Acinetobacter baumannii at Mulago Hospital in Kampala, Uganda (2007-2009). Springerplus 2016; 5: 1308. doi: 10.1186/ s40064-016-2986-7. eCollection 2016.

37. Chandel A, Raza Mohammad S. Antimicrobial susceptibility patterns of Pseudomonas aeruginosa clinical isolates at a tertiary care hospital in Kathmandu, Nepal. Asian J Pharm Clin Res 2013; 6: 235-58. 